\title{
Finite-Time Convergent Observer for A Class of Nonlinear Systems Using Homogeneous Method
}

\author{
Weisong Tian, Chunjiang Qian and Michael T. Frye
}

\begin{abstract}
This paper develops nonlinear observers by using the newly developed homogeneous observer for a class of lowertriangular nonlinear systems whose solution trajectories are bounded. We show that the estimated states of the proposed observer can converge to the real states in a finite time for small initial estimation errors. We also show that by adjusting the observer gain the required range of the initial estimation errors can be expanded.
\end{abstract}

\section{INTRODUCTION}

The objective of this paper is to design a finite-time convergent state observer for a class of nonlinear systems described by:

$$
\begin{aligned}
\dot{x}_{1} & =x_{2}+f_{1}\left(x_{1}\right) \\
\dot{x}_{2} & =x_{3}+f_{2}\left(x_{1}, x_{2}\right) \\
& \vdots \\
\dot{x}_{n-1} & =x_{n}+f_{n-1}\left(x_{1}, x_{2}, \cdots, x_{n-1}\right) \\
\dot{x}_{n} & =f_{n}\left(x_{1}, x_{2}, \cdots, x_{n}\right) \\
y & =x_{1}
\end{aligned}
$$

where $y \in \mathbb{R}$ is the system output. The nonlinear functions $f_{i}=f_{i}\left(x_{1}, \cdots, x_{i}\right)$ for $i=1,2, \cdots, n$, are $\mathcal{C}^{1}$ (continuously differentiable).

The problem of estimating the unmeasurable states of a nonlinear system from its output has been receiving a great deal of attention and there are quite a number of early works have been devoted. One existing method is using linearization of the system [3], [9], [11]. In addition, a locally convergent nonlinear observer by using back-stepping method is constructed in [10]. The estimation error goes to zero when the initial value of estimation error is not too large. This method can be easily implemented because of its recursive algorithm. A high gain observer introduced in [5] is another observer designing method for nonlinear systems. The paper [12] introduced a high gain observer for systems with bounded solutions.

More recently, new design methods based on homogeneous systems theory are developed to construct homogeneous observers. The work [14] introduced a nonlinear observer with a homogeneous structure even for a linear system. The new construction of the observer enables us to relax the long-existing linear growth condition used for nonlinear

Weisong Tian (E-mail: weisong.tian@ gmail.com) and Chunjiang Qian (Email: chunjiang.qian@utsa.edu) are with the Department of Electrical and Computer Engineering, University of Texas at San Antonio, San Antonio, TX, USA.

Michael T. Frye (E-mail: mfrye@uiwtx.edu) is with the Department of Engineering, University of the Incarnate Word, San Antonio, TX, USA observer design. Later, the papers [1], [13] extended the homogeneous observer introduced in [14] to more general nonlinear systems. In addition, the observers constructed in the papers [13] and [15] have a fast convergence rate, i.e. the estimates will converge to the original states in a finite time, rather than asymptotically. However, to achieve this, various conditions have been imposed on the nonlinearities.

In this paper, we are also interested in designing a finitetime homogeneous observer for a class of lower-triangular nonlinear systems. Instead of pursuing global finite-time convergence, we will prove the estimated states are finitetime convergent to the real states of system (1.1) when the initial errors are small. By pursuing this less ambitious goal, we can show that there is no need of growth conditions imposed on the nonlinearities [13] and [15]. In addition, we will also prove that under the assumption of bounded solution trajectories, the requirement for small initial condition can be lifted by introducing and adjusting a scaling gain in the homogeneous observer.

The rest of this paper is organized as follows: In Section II, we introduce some useful lemmas and existing homogeneous design methods. In Section III, we construct a low-order homogeneous observer for lower-triangular systems and we prove it is finite-time convergent when the initial error is small. Several illustrative examples are discussed in this section. Our conclusion is included in Section IV.

\section{Preliminaries of Homogeneous Observers}

In this section, we introduce some useful definitions and lemmas from homogeneous system theory which will be constantly used in proving the main results. The innovative idea of homogeneity was introduced for the stability analysis of a nonlinear system [6] and has led to a number of interesting results. Listed below are the definitions of homogeneous functions and homogeneous systems with weighted dilation (refer to References [8], [4], [7], [2] for details).

Weighted homogeneity: For fixed coordinates $\left(x_{1}, \cdots, x_{n}\right) \in \mathbb{R}^{n}$ and real numbers $r_{i}>0$, for $i=1,2, \cdots, n$,

- the dilation $\Delta_{\varepsilon}(x)=\left(\varepsilon^{r_{1}} x_{1}, \cdots, \varepsilon^{r_{n}} x_{n}\right), \forall \varepsilon>0$, with $r_{i}$ being called as the weights of the coordinates.

- a function $V \in C\left(\mathbb{R}^{n}, \mathbb{R}\right)$ is said to be homogeneous of degree $\tau$ if there is a real number $\tau \in \mathbb{R}$ such that

$$
\forall x \in \mathbb{R}^{n} \backslash\{0\}, \varepsilon>0, V\left(\Delta_{\varepsilon}(x)\right)=\varepsilon^{\tau} V\left(x_{1}, x_{2}, \cdots, x_{n}\right) .
$$

- a function $f: \mathbb{R}^{n} \rightarrow \mathbb{R}^{n}$ is said to be homogeneous if there exists $\left(r_{1}, r_{2}, \cdots, r_{n}\right) \in((0,+\infty))^{n}$ and $\tau \in \mathbb{R}$ such 
that $\forall x=\left(x_{1}, x_{2}, \cdots, x_{n}\right)^{T} \in \mathbb{R}^{n} \forall \varepsilon>0$, and

$$
f_{i}\left(\varepsilon^{r_{1}} x_{1}, \cdots, \varepsilon^{r_{n}} x_{n}\right)=\varepsilon^{\tau+r_{i}} f_{i}(x), i=1, \cdots, n .
$$

- a vector $V \in C\left(\mathbb{R}^{n}, \mathbb{R}^{n}\right)$ is said to be homogeneous of degree $\tau$ if there is a real number $\tau \in \mathbb{R}$ such that for $i=1,2, \cdots, n$

$\forall x \in \mathbb{R}^{n} \backslash\{0\}, \varepsilon>0, f_{i}\left(\Delta_{\varepsilon}(x)\right)=\varepsilon^{\tau+r_{i}} V\left(x_{1}, x_{2}, \cdots, x_{n}\right)$.

- a homogeneous $p$-norm is defined as

$$
\|x\|_{\Delta, p}=\left(\sum_{i=1}^{n}\left|x_{i}\right|^{\frac{p}{r_{i}}}\right)^{\frac{1}{p}}, \forall x \in \mathbb{R}^{n}
$$

for a constant $p \geq 1$. For the simplicity, in this paper, we choose $p=2$, and write $\|x\|_{\Delta}$ for $\|x\|_{\Delta, 2}$.

In what follows, we list some useful properties of homogeneous systems.

Lemma 2.1: If the trivial solution $x=0$ of the $\Delta_{\varepsilon}$ homogeneous system

$$
\dot{x}=f(x), f(0)=0
$$

is globally stable, there exists a $\Delta_{\varepsilon}$-homogeneous Lyapunov function $V$, which is positive definite and proper, such that

$$
\dot{V}=\frac{\partial V}{\partial x} f(x)<0, \forall x \neq 0 .
$$

Lemma 2.2: Given a dilation weight $\Delta=$ $\left(r_{1}, r_{2}, \cdots, r_{n}\right)$, suppose $V_{1}(x)$ and $V_{2}(x)$ are respectively homogeneous functions of degree $\tau_{1}$ and $\tau_{2}$. Then $V_{1}(x) V_{2}(x)$ is also homogeneous with respect to the same dilation weight $\Delta$. Moreover, the homogeneous degree of $V_{1}(x) V_{2}(x)$ is $\tau_{1}+\tau_{2}$

Lemma 2.3: Assume $V: \mathbb{R}^{n} \rightarrow \mathbb{R}$ is a homogeneous function of degree $\tau$ with respect to the dilation weight $\Delta$. Then the following holds: (A) $\frac{\partial V}{\partial x_{i}}$ is homogeneous of degree $\tau-r_{i}$ with $r_{i}$ being the homogeneous weight of $x_{i}$. (B) There is a constant $\bar{c}$ such that

$$
V(x) \leq \bar{c}\|x\|_{\Delta}^{\tau}
$$

Moreover, if $V(x)$ is positive definite, $\underline{c}\|x\|_{\Delta}^{\tau} \leq V(x)$ for a constant $\underline{c}>0$.

Next we review an existing result on homogeneous observers. The paper [14] introduced a homogeneous observer estimating the nonlinear parts of unmeasurable states. In what follows, we list the homogeneous observer for the following system:

$$
\begin{gathered}
\dot{x}_{1}=x_{2} \\
\vdots \\
\dot{x}_{n-1}=x_{n} \\
\dot{x}_{n}=u \\
y=x_{1} .
\end{gathered}
$$

The low-order observer for system (2.2) is constructed in the form of:

$$
\begin{aligned}
\dot{\hat{x}}_{1} & =\hat{x}_{2}+c_{1}\left(x_{1}-\hat{x}_{1}\right)^{r_{2}} \\
\dot{\hat{x}}_{2} & =\hat{x}_{3}+c_{2}\left(x_{1}-\hat{x}_{1}\right)^{r_{3}} \\
\vdots & \\
\dot{\hat{x}}_{n-1} & =\hat{x}_{n}+c_{n-1}\left(x_{1}-\hat{x}_{1}\right)^{r_{n}} \\
\dot{\hat{x}}_{n} & =u+c_{n}\left(x_{1}-\hat{x}_{1}\right)^{r_{n+1}}
\end{aligned}
$$

where $r_{1}=1$. For $i=1,2, \cdots, n$ and $-1 / n<\tau_{1}<0$, $r_{i+1}=r_{i}+\tau_{1}$, and $c_{i}$ are appropriately chosen constants Define $e_{i}=x_{i}-\hat{x}_{i}, i=1,2, \cdots, n$, then we obtain the error dynamics as follows:

$$
\begin{aligned}
\dot{e}_{1} & =e_{2}-c_{1}\left(x_{1}-\hat{x}_{1}\right)^{r_{2}} \\
\dot{e}_{2} & =e_{3}-c_{2}\left(x_{1}-\hat{x}_{1}\right)^{r_{3}} \\
\vdots & \\
\dot{e}_{n-1} & =e_{n}-c_{n-1}\left(x_{1}-\hat{x}_{1}\right)^{r_{n}} \\
\dot{e}_{n} & =-c_{n}\left(x_{1}-\hat{x}_{1}\right)^{r_{n+1}} .
\end{aligned}
$$

It was shown in [14] that the above error dynamic is globally asymptotic stable for appropriate constants $c_{i}$ 's.

\section{HOMOGENEOUS OBSERVER FOR LOWER-TRIANGULAR SYSTEMS}

In this section, we show that the homogeneous observer introduced in [14] can be applied to estimate states of lowertriangular nonlinear systems. First we review an existed back-stepping method. The paper [10] introduced an observer using a back-stepping method, which is applicable to a class of smooth nonlinear systems in the following form:

$$
\begin{gathered}
\dot{x}_{1}=x_{2} \\
\vdots \\
\dot{x}_{n-1}=x_{n} \\
\dot{x}_{n}=f_{n}(x) \\
y=x_{1}
\end{gathered}
$$

where $f_{n}(x)$ is a smooth function with $f_{n}(0)=0$.

The observer using back-stepping method is in the following form:

$$
\begin{aligned}
\dot{\hat{x}}_{1} & =\hat{x}_{2}+\varphi_{1}(\hat{x})\left(x_{1}-\hat{x}_{1}\right) \\
\dot{\hat{x}}_{2} & =\hat{x}_{3}+\varphi_{2}(\hat{x})\left(x_{1}-\hat{x}_{1}\right) \\
\vdots & \\
\dot{\hat{x}}_{n-1} & =\hat{x}_{n}+\varphi_{n-1}(\hat{x})\left(x_{1}-\hat{x}_{1}\right) \\
\dot{\hat{x}}_{n} & =f_{n}(\hat{x})+\varphi_{n}(\hat{x})\left(x_{1}-\hat{x}_{1}\right)
\end{aligned}
$$

where gain functions $\varphi_{i}, i=1, \cdots, n$ are computed in the 
following way:

$$
\begin{aligned}
\varphi_{1}(\hat{x}) & =b_{n+1, n}(\hat{x})+f_{n ; n}(\hat{x}) \\
\varphi_{2}(\hat{x}) & =b_{n+1, n-1}(\hat{x})+f_{n ; n-1}(\hat{x}) \\
& \vdots \\
\varphi_{n-1}(\hat{x}) & =b_{n+1,2}(\hat{x})+f_{n ; 2}(\hat{x}) \\
\varphi_{n}(\hat{x}) & =b_{n+1,1}(\hat{x})+f_{n ; 1}(\hat{x})
\end{aligned}
$$

with $f_{n ; i}=\frac{\partial f_{n}}{\partial x_{i}}(\hat{x}), i=1, \cdots, n$ and

$$
\begin{aligned}
b_{2,1}= & c_{1} \\
b_{i, 1}= & b_{i-2,1}-\varphi_{i-3}+c_{i-1}\left(b_{i-1,1}-\varphi_{i-2}\right) \\
& +\left(b_{i-1,1}-\varphi_{i-2}\right)^{\prime}-\sum_{j=1}^{i-2}\left(b_{i-1, j}-\varphi_{i-j-1}\right) \varphi_{j} \\
b_{i, j}= & b_{i-2, j}-\varphi_{i-j-2}+c_{i-1}\left(b_{i-1, j}-\varphi_{i-j-1}\right) \\
& +\left(b_{i-1, j}-\varphi_{i-j-1}\right)^{\prime}-b_{i-1, j-1} \\
b_{i, i-2}= & +c_{i-1}\left(b_{i-1, i-2}-\varphi_{1}\right)+\left(b_{i-1, i-2}-\varphi_{1}\right)^{\prime}-b_{i-1, j-3} \\
b_{i, i-1}= & c_{i-1}+b_{i-1, i-2} .
\end{aligned}
$$

Defining $e_{i}=x_{i}-\hat{x}_{i}$, for $i=1,2, \cdots, n$, the error dynamics are presented as following:

$$
\begin{aligned}
\dot{e}_{1} & =e_{2}-\varphi_{1}(\hat{x}) e_{1} \\
\dot{e}_{2} & =e_{3}-\varphi_{2}(\hat{x}) e_{1} \\
\vdots & \\
\dot{e}_{n-1} & =e_{n}-\varphi_{n-1}(\hat{x}) e_{1} \\
\dot{e}_{n} & =f_{n}(x)-f_{n}(\hat{x})-\varphi_{n}(\hat{x}) e_{1} .
\end{aligned}
$$

According to the results in [10], with the gain functions $\varphi_{n}(\hat{x})$ for $1 \leq i \leq n$ selected as (3.7)-(3.8), $e(t) \rightarrow 0$ as $t \rightarrow \infty$ for small $e_{i}(0)$ 's.

The method introduced in [10] is based on back-stepping, and it is locally convergent (refer to [10] for proof detail). This method is easily implemented because of its recursive algorithm, and it has good accuracy of estimating.

As shown in [10], this observer is locally convergent, which requires the initial errors $e_{i}(0)=x_{i}(0)-\hat{x}_{i}(0)$ to be small. Moreover, the functions $\varphi_{i}(\hat{x})$ are quite complicated. Now we provide an alternative solution based on homogeneous observer without computing $\varphi_{i}(\hat{x})$.

Consider the lower-triangular system in the form of (1.1). Based on the homogeneous observer design method we introduced in the previous section, we can construct a loworder homogeneous observer for (1.1)

$$
\begin{aligned}
\dot{\hat{x}}_{1} & =\hat{x}_{2}+f_{1}\left(x_{1}\right)+c_{1} e_{1}^{r_{2}} \\
\dot{\hat{x}}_{2} & =\hat{x}_{3}+f_{2}\left(x_{1}, \hat{x}_{2}\right)+c_{2} e_{1}^{r_{3}} \\
\vdots & \\
\dot{\hat{x}}_{n-1} & =\hat{x}_{n}+f_{n-1}\left(x_{1}, \hat{x}_{2}, \cdots, \hat{x}_{n-1}\right)+c_{n-1} e_{1}^{r_{n}} \\
\dot{\hat{x}}_{n} & =f_{n}\left(x_{1}, \hat{x}_{2}, \cdots, \hat{x}_{n}\right)+c_{n} e_{1}^{r_{n+1}}
\end{aligned}
$$

where constants $c_{i}$ and $r_{i}$ are defined in (2.3).
By defining the error as $e_{i}=x_{i}-\hat{x}_{i}$, we can obtain the error dynamics.

$$
\begin{aligned}
\dot{e}_{1}= & e_{2}-c_{1} e_{1}^{r_{2}}+f_{1}\left(x_{1}\right)-f_{1}\left(x_{1}\right) \\
\dot{e}_{2}= & e_{3}-c_{2} e_{1}^{r_{3}}+f_{2}\left(x_{1}, x_{2}\right)+f_{2}\left(x_{1}, \hat{x}_{2}\right) \\
& \vdots \\
\dot{e}_{n-1}= & e_{n}-c_{n-1} e_{1}^{r_{n}}+f_{n-1}\left(x_{1}, x_{2}, \cdots, x_{n-1}\right) \\
& -f_{n-1}\left(x_{1}, \hat{x}_{2}, \cdots, \hat{x}_{n-1}\right) \\
\dot{e}_{n}= & -c_{n} e_{1}^{r_{n+1}}+f_{n}\left(x_{1}, x_{2}, \cdots, x_{n}\right)-f_{n}\left(x_{1}, \hat{x}_{2}, \cdots, \hat{x}_{n}\right) .
\end{aligned}
$$

Theorem 3.1: Suppose that $K$ is a compact and positively invariant set for system (1.1). For the initial condition $\hat{x}(0)$ of equation (3.10) close to $x(0)$ in (1.1), the error dynamics (3.11) are finite-time stable.

Proof. The error dynamics can be written as:

$$
\begin{gathered}
\dot{e}=\left(\begin{array}{c}
\dot{e}_{1} \\
\dot{e}_{2} \\
\vdots \\
\dot{e}_{n-1} \\
\dot{e}_{n}
\end{array}\right)=\left(\begin{array}{c}
e_{2}-c_{1} e_{1}^{r_{2}} \\
e_{3}-c_{2} e_{1}^{r_{3}} \\
\vdots \\
e_{n}-c_{n-1} e_{1}^{r_{n}} \\
-c_{n} e_{1}^{r_{n+1}}
\end{array}\right) \\
+\left(\begin{array}{c}
f_{1}\left(x_{1}\right)-f_{1}\left(x_{1}\right) \\
f_{2}\left(x_{1}, x_{2}\right)-f_{2}\left(x_{1}, \hat{x}_{2}\right) \\
\vdots \\
f_{n-1}\left(x_{1}, x_{2}, \cdots, x_{n-1}\right)-f_{n-1}\left(x_{1}, \hat{x}_{2}, \cdots, \hat{x}_{n-1}\right) \\
f_{n}\left(x_{1}, x_{2}, \cdots, x_{n}\right)-f_{n}\left(x_{1}, \hat{x}_{2}, \cdots, \hat{x}_{n}\right)
\end{array}\right) .
\end{gathered}
$$

For $i=1,2, \cdots, n$, by the continuous differentiability of the functions $f_{i}$ 's and the mean value theorem, we have the following for small error $\|e\|$ :

$$
\begin{aligned}
& \left|f_{i}\left(x_{1}, x_{2}, \cdots, x_{i}\right)-f_{i}\left(x_{1}, \hat{x}_{2}, \cdots, \hat{x}_{i}\right)\right| \\
& \leq H_{i}\left(x_{1}, x_{2}, \cdots, x_{i}, \hat{x}_{2}, \cdots, \hat{x}_{i}\right)\left(\left|e_{2}\right|+\cdots+\left|e_{i}\right|\right) \\
& \leq \tilde{H}_{i}\left(x_{1}, \cdots, x_{i}\right)\left(\left|e_{2}\right|+\cdots+\left|e_{i}\right|\right)
\end{aligned}
$$

where $\tilde{H}_{i}$ are smooth functions for $i=1,2, \cdots, n$. For our low-order observer, noting that $0<r_{i}<r_{i-1}<1$ for $i=$ $1,2, \cdots, i$, one obtains $\frac{r_{i+1}}{r_{2}}<1, \cdots, \frac{r_{i+1}}{r_{i}}<1$. Thus, here we can use this property to lead to the following equations:

$$
\begin{aligned}
& \left|f_{i}\left(x_{1}, x_{2}, \cdots, x_{n}\right)-f_{i}\left(x_{1}, \hat{x}_{2}, \cdots, \hat{x}_{n}\right)\right| \\
& =\tilde{H}_{i}\left(x_{1}, \cdots, x_{i}\right) \times\left(\left|e_{2}\right|^{\frac{r_{i+1}}{r_{2}}} \cdot\left|e_{2}\right|^{1-\frac{r_{i+1}}{r_{2}}}+\cdots\right. \\
& \left.+\left|e_{i}\right|^{\frac{r_{i+1}}{r_{i}}} \cdot\left|e_{i}\right|^{1-\frac{r_{i+1}}{r_{i}}}\right) \\
& \leq \tilde{H}_{i}\left(x_{1}, \cdots, x_{i}\right) \times\left(\left|e_{2}\right|^{\frac{r_{i+1}}{r_{2}}}+\cdots+\left|e_{i}\right|^{\frac{r_{i+1}}{r_{i}}}\right) \\
& \times\left(\left|e_{2}\right|^{1-\frac{r_{i+1}}{r_{2}}}+\cdots+\left|e_{i}\right|^{1-\frac{r_{i+1}}{r_{i}}}\right) .
\end{aligned}
$$

It has been proved in [14] that there are constants $c_{i}$, for $i=1, \cdots, n$, such that the homogeneous truncated system 
of (3.12), i.e.

$$
\dot{e}=\left(\begin{array}{c}
e_{2}-c_{1} e_{1}^{r_{2}} \\
e_{3}-c_{2} e_{1}^{r_{3}} \\
\vdots \\
e_{n}-c_{n-1} e_{1}^{r_{n}} \\
-c_{n} e_{1}^{r_{n+1}}
\end{array}\right)
$$

is finite-time stable. By using Lemma 2.1, we can always find a homogeneous Lyapunov function $V$ with degree $k$, which makes the above truncated system satisfy the following relation:

$$
\frac{\partial V}{\partial e}\left(\begin{array}{c}
e_{2}-c_{1} e_{1}^{r_{2}} \\
e_{3}-c_{2} e_{1}^{r_{3}} \\
\vdots \\
e_{n}-c_{n-1} e_{1}^{r_{n}} \\
-c_{n} e_{1}^{r_{n+1}}
\end{array}\right)=-\omega(e)
$$

where $\omega(e)$ is a homogeneous function of degree $k+\tau_{1}$ and a positive definite function.

Combining (3.15) and (3.14), the derivative of the homogeneous Lyapunov function along the system (3.12) is

$$
\begin{aligned}
\left.\dot{V}\right|_{(3.12)} \leq & -\omega(e)+\sum_{i=2}^{n}\left|\frac{\partial V}{\partial e_{i}}\right|\left(\left|e_{2}\right|^{\frac{r_{i+1}}{r_{2}}}+\cdots+\left|e_{i}\right|^{\frac{r_{i+1}}{r_{i}}}\right) \\
& \times \tilde{H}_{i}(\cdot)\left[\left|e_{2}\right|^{1-\frac{r_{i+1}}{r_{2}}}+\cdots+\left|e_{n}\right|^{1-\frac{r_{i+1}}{r_{i}}}\right] .
\end{aligned}
$$

On the other hand, by Lemma 2.2,

$$
\left|\frac{\partial V}{\partial e_{i}}\right|\left(\left|e_{2}\right|^{\frac{r_{i+1}}{r_{2}}}+\cdots+\left|e_{i}\right|^{\frac{r_{i+1}}{r_{i}}}\right)
$$

is a homogeneous term with degree of $k+\tau_{1}$. Thus, by Lemma 2.3, we can find a constant $c$ such that

$$
\left|\frac{\partial V}{\partial e_{i}}\right|\left(\left|e_{2}\right|^{\frac{r_{i+1}}{r_{2}}}+\cdots+\left|e_{i}\right|^{\frac{r_{i+1}}{r_{i}}}\right) \leq c \cdot \omega(e),
$$

for the positive definite homogeneous term $\omega(e)$.

Substituting (3.18) into (3.16) yields

$$
\begin{aligned}
\left.\dot{V}\right|_{(3.12)} \leq & -\omega(e)\left[1-c \sum_{i=2}^{n} \tilde{H}_{i}\left(x_{1}, \cdots, x_{i}\right)\right. \\
& \left.\times\left(\left|e_{2}\right|^{1-\frac{r_{i+1}}{r_{2}}}+\cdots+\left|e_{i}\right|^{1-\frac{r_{i+1}}{r_{i}}}\right)\right] .
\end{aligned}
$$

Since $0<r_{n+1}<r_{n}<\cdots<r_{2}<r_{1}=1$, we have $0<\frac{r_{i+1}}{r_{i}}<1$, for $i=1, \cdots, n$, and the power $1-\frac{r_{i+1}}{r_{i}}>0$ for $i=1, \cdots, n$. Consequently, when the error $e_{i}$ is small enough, the term

$$
c \sum_{i=2}^{n} \tilde{H}_{i}\left(x_{1}, \cdots, x_{i}\right)\left(\left|e_{2}\right|^{1-\frac{r_{i+1}}{r_{2}}}+\cdots+\left|e_{i}\right|^{1-\frac{r_{i+1}}{r_{i}}}\right)<1,
$$

for bounded $x$ in $K$. Clearly, we have proven (3.19) is negative definite due to the boundedness of $x$, and we can conclude that the error dynamics (3.11) is finite-time stable as long as $\|e\|$ is small enough.
Example 3.1: Let's consider the example studied in [10] using back-stepping observer:

$$
\begin{aligned}
\dot{x}_{1} & =2 x_{2} \\
\dot{x}_{2} & =2 x_{1}-3 x_{1}^{2}-x_{2}\left(x_{1}^{3}-x_{1}^{2}+x_{2}^{2}-\mu\right) \\
y & =x_{1},
\end{aligned}
$$

where $\mu$ is a negative constant. This system is known as homoclinic loop. According to our previous discursion, we can construct a low-order observer as following:

$$
\begin{aligned}
\tau_{1} & =-\frac{2}{9} \\
\Rightarrow r_{2} & =r_{1}+\tau_{1}=1-\frac{2}{9}=\frac{7}{9} \\
\Rightarrow r_{3} & =r_{2}+\tau_{1}=\frac{7}{9}-\frac{2}{9}=\frac{5}{9} .
\end{aligned}
$$

Thus our low-order observer is

$$
\begin{aligned}
& \dot{\hat{x}}_{1}=2 \hat{x}_{2}+e_{1}^{\frac{7}{9}} \\
& \dot{\hat{x}}_{2}=2 y-3 y^{2}-\hat{x}_{2}\left(y^{3}-y^{2}+\hat{x}_{2}^{2}-\mu\right)+e_{1}^{\frac{5}{9}} .
\end{aligned}
$$

In order to demonstrate the performance of our design, we provide the simulations with different initial values.

In Fig.1, we choose initial errors $x(0)=\left(\begin{array}{l}0.1 \\ 0.1\end{array}\right)$ and $\hat{x}(0)=\left(\begin{array}{l}1.0 \\ 1.0\end{array}\right)$, but both initial states and estimation values are relatively small. Our low-order homogeneous observer still have outstanding accuracy convergence rate.

In Fig.2, we further show the performance of convergence rate of our low-order observer with larger initial values but small initial errors $x(0)=\left(\begin{array}{c}2.0 \\ 2.0\end{array}\right)$ and $\hat{x}(0)=\left(\begin{array}{l}2.1 \\ 1.9\end{array}\right)$, we can learn that the convergence rate is still acceptable. This example shows our finite-time observer only asks for small initial estimation error, but the states do not have to be bounded in a small neighborhood of the origin.

So far, we have designed an observer which is convergent if the initial errors between the observer states and real system states are sufficiently small. In what follows, we will show that this small initial condition can be relaxed by designing a new observer which integrates the technique introduced in [12].

First, we define a unit saturation function $\operatorname{sat}(s)$ as

$$
\operatorname{sat}(s)= \begin{cases}1, & \text { for } s \geq 1 \\ s, & \text { for }-1<s<1 \\ -1, & \text { for } s \leq-1\end{cases}
$$

Lemma 3.1: [12] Given real numbers $s_{1}, s_{2}$ and $m>0$, suppose that $\left|s_{1}\right| \leq m$. Then,

$$
\left|s_{1}-m \cdot \operatorname{sat}\left(\frac{s_{2}}{m}\right)\right| \leq\left|s_{1}-s_{2}\right| .
$$



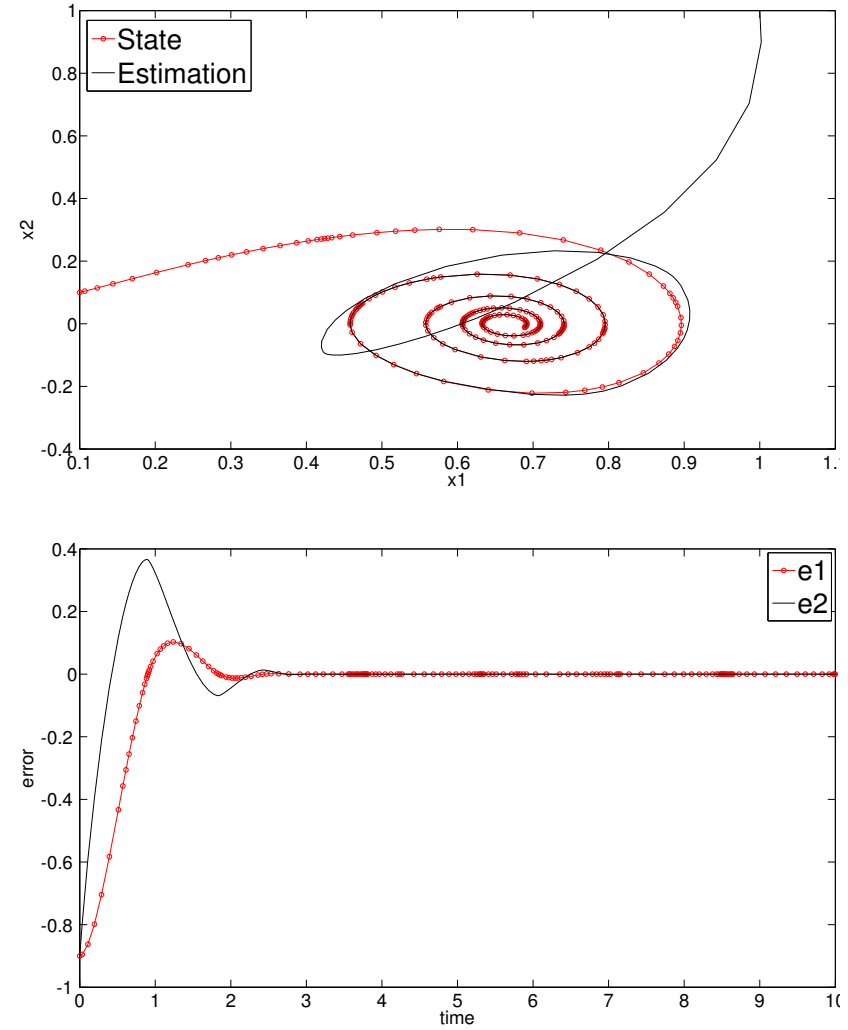

Fig. 1. Estimation and error dynamics inside the homoclinic loop with small initial state but big initial errors.

By using Lemma 2.5 and Claim 1 from [12], we can construct a homogeneous observer as following:

$$
\begin{aligned}
\dot{\hat{x}}_{1} & =\hat{x}_{2}+f_{1}\left(\operatorname{sat}_{N}\left(\hat{x}_{1}\right)\right)+L c_{1} e_{1}^{r_{2}} \\
\dot{\hat{x}}_{2} & =\hat{x}_{3}+f_{2}\left(\operatorname{sat}_{N}\left(\hat{x}_{1}\right), \operatorname{sat}_{N}\left(\hat{x}_{2}\right)\right)+L^{2} c_{2} e_{1}^{r_{3}} \\
\quad & \\
\dot{\hat{x}}_{n-1} & =\hat{x}_{n}+f_{n-1}\left(\operatorname{sat}_{N}\left(\hat{x}_{1}\right), \cdots, \operatorname{sat}_{N}\left(\hat{x}_{n-1}\right)\right)+L^{n-1} c_{n-1} e_{1}^{r_{n}} \\
\dot{\hat{x}}_{n} & =f_{n}\left(\operatorname{sat}_{N}\left(\hat{x}_{1}\right), \cdots, \operatorname{sat}_{N}\left(\hat{x}_{n}\right)\right)+L^{n} c_{n} e_{1}^{r_{n+1}}
\end{aligned}
$$

where the gain $L$ is a constant greater or equal than 1 , the bound $N \geq\left|x_{i}(t)\right|$, and $\operatorname{sat}_{N}(x)=N \cdot \operatorname{sat}\left(\frac{x}{N}\right)$.

By defining a new error term $\varepsilon_{i}=e_{i} / L^{i-1}$, the new error dynamic is:

$$
\begin{gathered}
\dot{\varepsilon}=\left(\begin{array}{c}
\dot{\varepsilon}_{1} \\
\dot{\varepsilon}_{2} \\
\vdots \\
\dot{\varepsilon}_{n-1} \\
\dot{\varepsilon}_{n}
\end{array}\right)=L\left(\begin{array}{c}
\varepsilon_{2}-c_{1} \varepsilon_{1}^{r_{2}} \\
\varepsilon_{3}-c_{2} \varepsilon_{1}^{r_{3}} \\
\vdots \\
\varepsilon_{n}-c_{n-1} \varepsilon_{1}^{r_{n}} \\
-c_{n} \varepsilon_{1}^{r_{n+1}}
\end{array}\right) \\
+\left(\begin{array}{c}
\left.f_{1}\right)-f_{1}\left(\operatorname{sat}_{N}\left(\hat{x}_{1}\right)\right) \\
\left(f_{2}\left(x_{1}, x_{2}\right)-f_{2}\left(\operatorname{sat}_{N}\left(\hat{x}_{1}\right), \operatorname{sat}_{N}\left(\hat{x}_{2}\right)\right)\right) / L \\
\vdots \\
\left(f_{n-1}\left(x_{1}, \cdots, x_{n-1}\right)-f_{n-1}\left(\operatorname{sat}_{N}\left(\hat{x}_{1}\right), \cdots, s_{N}\left(\hat{x}_{n-1}\right)\right)\right) / L^{n-2} \\
\left(f_{n}\left(x_{1}, \cdots, x_{n}\right)-f_{n}\left(\operatorname{sat}_{N}\left(\hat{x}_{1}\right), \cdots, \operatorname{sat}_{N}\left(\hat{x}_{n-1}\right)\right)\right) / L^{n-1}
\end{array}\right)
\end{gathered}
$$
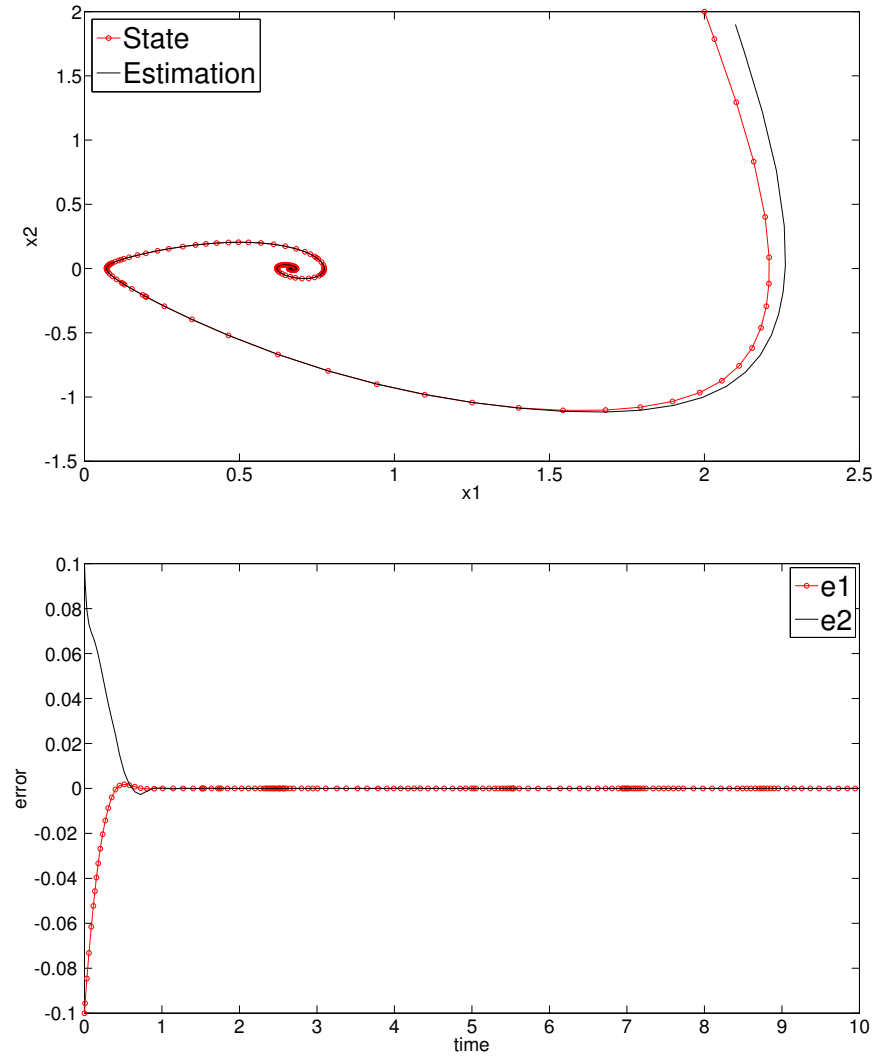

Fig. 2. Estimation and error dynamics inside the homoclinic loop with big initial states and small initial errors.

With the help of Lemma 3.1, similar to the proof of Theorem 3.1, it can be proven that when the solution trajectories are bounded, the error dynamics (3.27) is finite-time stable, for a large enough $L$. This method significantly enlarges the accepted range initial estimation, and the observer can still be finite-time stable by adjusting $L$.

In what follows, we apply observer (3.26) to system (3.21). We choose the initial states the same as those used in Fig.1, and the constants $L$ and $N$ are chosen as 5 and 2, respectively. The simulation is presented in Fig.3.

\section{CONCLUSIONS}

In this paper, for a class of lower-triangular nonlinear systems, we designed a homogeneous observer which is finite-time convergent when the initial estimation error is not too large. Then we integrate our design with the saturation method introduced in [12] to show that under the condition of bounded solution trajectories, the new observer has the property of finite time convergence, if the constant gain $L$ is large enough.

\section{ACKNOWLEDGEMENT}

This work is supported in part by U.S. National Science Foundation under Grant No. HRD-0932339, and the Collaborative Research Seed Grant from the University of Texas at San Antonio. 

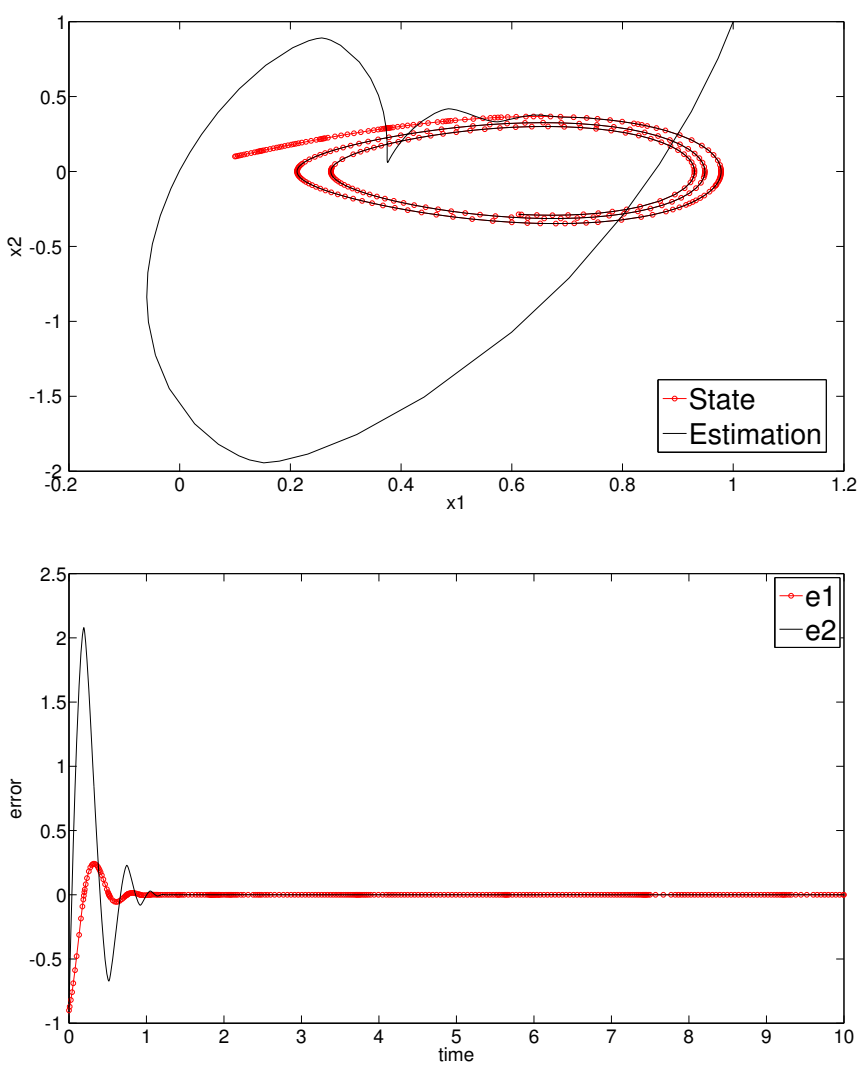

Fig. 3. Estimation and error dynamics inside the homoclinic by using observer (3.26)

\section{REFERENCES}

[1] V. Andrieu, L. Praly, and A. Astolfi. High gain observers with updated gain and homogeneous correction term. Automatica, 45(2):422-428, February 2009.

[2] A. Bacciotti and L. Roiser. Liapunov functions and stability in control theory. In Lecture Notes in Control and Information Sciences, volume 267. Springer: Berlin, 2001.

[3] D. Bestle and M. Zeitz. Canonical form observer design for non-linear time-variable system. International Journal of Control, 38(2):419-431, August 1983.

[4] W. Dayawansa, C. Martin, and G. Knowles. Asymptotic stabilization of a class of smooth two dimensional systems. SIAM Journal on Control and Optimization, 28(6):1321-1349, 1990.

[5] J. P. Gauthier, H. Hammouri, and S. Othman. A simple observer for nonlinear systems, applications to bioreactors. IEEE TRANSACTIONS ON AUTOMATIC CONTROL, 37(6):875C880, 1992.

[6] W. Hann. Stability of motion. Springer-Verlag, 1967.

[7] H. Hermes. Homogeneous coordinates and continuous asymptotically stabilizing feedback controls. In S. Elaydi, editor, Differential Equations, Stability and Control, pages 249-260. Dekker: New York, 1991.

[8] M. Kawski. Homogeneous stabilizing feedback laws. Control Theory and Advanced Technology, 6(4):497-516, 1990.

[9] A. Krener and A. Isidori. Linearization by output injection and nonlinear observers. Systems and Control Letters, 3(1):47-52, 1983.

[10] A. Krener and W. Kang. Locally convergent nonlinear observers. SIAM J. Control Optim, 42(1):155-177, 2003.

[11] A. Krener and W. Respondek. Nonlinear observers with linearizable error dynamics. SIAM J. Control Optim., 23(2):197-216, 1985.

[12] H. Lei, J. Wei, and W. Lin. A global observer for obervable autonomous systems with bounded solution trajectories. In Proc. of the 44th IEEE Conference on Decision and Control, and the European Control Conference, pages 1911-1916, Seville, Spain, 2005.
[13] J. Li, C. Qian, and S. Ding. Global finite-time stabilization by output feedback for a class of uncertain nonlinear systems. International Journal of Control, 83(11):2241-2252, 2010.

[14] C. Qian. A homogeneous domination approach for global output feedback stabilization of a class of nonlinear systems. In Proceedings of 2005 American Control Conference, pages 4708-4715, June 2005.

[15] C. Qian and J. Li. Global output feedback stabilization of uppertriangular nonlinear systems using a homogeneous domination approach. International Journal of Robust and Nonlinear Control, 16(9):441-463, 2006 